\title{
REDES CONCEPTUALES: SU APLICACIÓN COMO INSTRUMENTO DIDÁCTICO EN TEMAS DE FÍSICA
}

\author{
GALAGOVSKY, L.R. ${ }^{1}$ y CILIBERTI, N. ${ }^{2}$ \\ 1 Secretaría Académica de la Facultad de Ciencias Exactas y Naturales. Universidad de \\ Buenos Aires. Ciudad Universitaria. 1428 Buenos Aires, Argentina. \\ 2 Instituto San Roque. Plaza 1160. 1427 Buenos Aires, Argentina.
}

\section{SUMMARY}

Concept Nets are the result of the need to acquire an instrument with which to improve the students' cognitive style of learning (Galagovsky 1993a).

For the last four years we have been researching about the benefits of its use in different subjects and levels. We now introduce an application in Physics, at High School level, developed during 1991-1992. A new research period 19931994 is being pursued.

\section{INTRODUCCIÓN}

Tradicionalmente los mapas conceptuales se han utilizado para desarrollar investigaciones sobre aprendizaje en temas de ciencias (Novack y Gowin 1988, Novack 1992, Moreira 1988, Moreira y Buchweitz 1987). Nuestro intento de utilizarlos como instrumento didáctico nos present 6 ciertas dificultades que pueden mirarse desde dos aspectos:

* Desde lo comunicacional:

Es muy difícil «leer» el mapa que ha construido otra persona porque, al no estar explicitadas todas las relaciones que unen conceptos, un docente puede interpretar dichos trazos de unión como correctos, cuando en realidad el sujeto que las construyó no era totalmente consciente de la relación precisa entre ambos nodos.

* Desde la estructura curricular de cada asignatura:

La jerarquía conceptual de los mapas traducida en una jerarquía gráfica vertical resulta confusa al tratar temas consecutivos en los cuales, obviamente, un concepto considerado más abarcativo dentro del primer tema puede ser jerárquicamente subordinado en el tema subsiguiente.
Estos inconvenientes nos condujeron a definir un nuevo instrumento gráfico-semántico: las redes conceptuales, según las siguientes consideraciones:

- Como recurso comunicacional, incluimos el concepto de oración nuclear', proveniente de la psicolinguiística (Chomsky 1972), y la necesidad de que cada relación entre nodos corresponda a la explicitacion de una de dichas estructuras gramaticales.

- Como recurso gráfico-conceptual, flexibilizamos su confección de tal forma que su disposición gráfica resulte independiente de la estructura jerárquica del tema.

Precisamos, entonces, las siguientes consignas para el armado de una red conceptual (Galagovsky 1993a):

a) Los nodos de la red serán ocupados por signos lingüísticos que representen conceptos esenciales del tema en cuestión.

b) La totalidad de las uniones que relacionan conceptos deberán exhibir leyendas que incluyan un verbo preciso, de tal forma que generen una oración nuclear entre nodos. 
c) Verbos tales como «afectan», "modifican», «está conectado con», etc. no son considerados precisos.

d) Las oraciones se leerán con un sentido señalado por una flecha. Necesariamente, la flecha inversa generará otra oración nuclear. No podrá construirse una oración que se extienda entre más de dos nodos.

e) La lectura de la red puede comenzar por cualquier nodo.

f) Se considerará artificial, la ordenación jerárquica de conceptos en relación con una disposición gráfica vertical. EI diseño gráfico sólo requerirá claridad para la lectura.

g) Se considerarán como conceptos fundamentales aquéllos a los que llegan y de los que parten la mayor cantidad de relaciones (flechas). Estos conceptos muy relacionados pueden ser, o no, los conceptos de jerarquía más abarcativa,

h) No se aceptatá la repetición de conceptos (nodos).

i) No se incluirán en las leyendas sobre las flechas conceptos que pertenezcan a la esencia del tema en cuestión y que no hayan sido previamente desglosados como nodos.

j) No se incluirán formulas matemáticas en los nodos, excepto en relaciones terminales con la leyenda: «se simboliza mediante».

Las redes conceptuales así definidas se muestran como excelente herramienta metodológica. Su confección nos impone representar las jerarquías conceptuales del modelo de aprendizaje significativo de D. Ausubel (Ausubel et al. 1978) mediante una red de análogos semán. ticos, a través de un ejercicio de metacognición permanente sobre los conceptos jerárquicamente organizados en nuestra estructura cognitiva.

\section{OBJETIVOS DEL PRESENTE TRABAJO}

Nuestro interés se centró en verificar si se produciría en el aprendizaje de los alumnos alguna modificación conceptual importante a partir del uso de este instrumento metodológico en el trabajo de clase.

La profesora Nora Ciliberti implementó este recurso didáctico en los momentos de cierre del tema cinemática en uno de sus dos cursos de tercer año en la asignatura Elementos de Físico-Química (bachillerato) habiendo enseñado desde un enfoque constructivista, en forma simétrica, en ambos cursos.

Los estimulantes resultados cualitativos obtenidos en el aprendizaje del tema cinemática (noviembre de 1991, Experiencia $)$ motivaron la extensión de las observaciones después del período de receso escolar (marzo de 1992, Experiencia II) y al tema de dinámica (abril-junio
1992, Experiencia $I I I)$, con el mismo grupo de alumnos que ya cursaban el cuarto año de la escuela secundaria.

\section{RELATO DE LA EXPERIENCIA, I: CON. FECCIÓN DE LA RED DE CINEMÁTICA}

\section{Las redes conceptuales confeccionadas previamente por las docentes}

Confeccionar una red conceptual exige al docente revisar, desde los conceptos, el tema en cuestión (Galagovsky et al. 1990, 1991). A veces, al hacer explícita la red, surge la evidencia de que los docentes hemos aprendido y enseñado reiteradamente el tema desde las definiciones, los algoritmos matemáticos de resolución y las reglas de aplicación.

La organización lógica del tema supone la construcción de nuevos conceptos sobre otros relacionados, que se deberían dominar (conceptos inclusores [Novack 1982]). Estos conceptos inclusores están en la estructura cognitiva del docente, quien a menudo «los utiliza» sin mencionarlos. Sin embargo, la no explicitación de dichos conceptos conduce, con frecuencia, a que permanezcan subyacentes o subsumidos (Novack 1992) para el entendimiento de los alumnos, y que por este motivo ellos no logren una construcción significativa del aprendizaje, que se compensa con esfuerzo memorístico, válido sólo en el corto plazo.

Construir la red conceptual es reconstruir explicitamente el tema desde los conceptos subyacentes hacia los conceptos nuevos; y esto, que nos parecía sencillo por haber enseñado el tema durante años, se nos revel 6 como un gran esfuerzo intelectual que nos demandó varias horas de discusión.

Para favorecer la claridad gráfica presentamos la red de cinemática que construimos subdividida en red \#1 y red $\# 2$ (ver página 348 ). Bucear en los errores frecuentes de los alumnos nos permitio descubrir algunos de dichos conceptos inclusores subyacentes, subsumidos, faltantes o mal entendidos. Estos errores comunes en el terna de cinemática, citados a continuación, se repiten año tras año, tanto en los alumnos del nivel secundario como los del primer ciclo universitario (ciclo básico común): * los alumnos confunden trayectoria con desplazamien-
to de un móvil.

También confunden trayectoria en un sistema de coordenadas cartesianas, con las curvas de los gráficos de $\mathrm{x}=\mathrm{f}(\mathrm{t})$, como se señala en la figura 1 . Incluimos, entonces, los conceptos de sistemas de referencia y sus relaciones, como se muestra en la red \#1 y el concepto de gráficos en la red $\# 2$.

* Los alumnos asocian una velocidad negativa con un móvil que frena. En ambas redes se analiza que el signo de la velocidad depende del sistema de referencia desde donde se miden las posiciones inicial y final; y surge el 


\section{Figura 1}
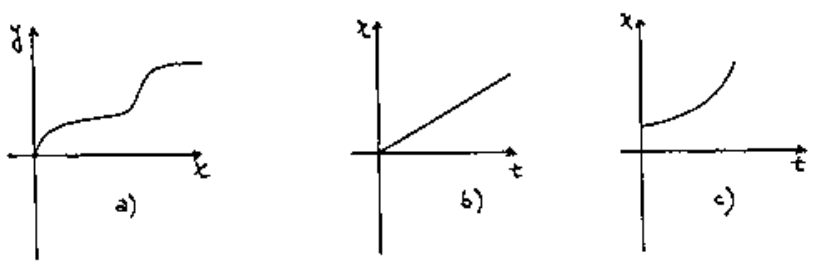

Los alumnos suelen confundir el significado de curvas como las siguientes:

a) Trayectoria de un móvil en el plano $x y$.

b) Curva de de un grafico $x=f(t)$ para movimiento uniforme.

c) Curva de un gráfico $\mathrm{x}=\mathrm{f}(\mathrm{t})$ para movimiento uniformemente variado.

carácter vectorial de la velociđad como consecuencia del carácter vectorial de la posición ${ }^{2}$.

\section{La elección del grupo de alumnos}

El tema de cinemática se đio como últímo bloque del currículo (septiembre-noviembre de 1991) a sendas divisiones de tercer año de bachillerato, grupos que denominaremos A y B. En ambos cursos se utilizó una idéntica planificación, textos, guías de problemas y recursos informáticos ${ }^{3}$, elaborados desde una perspectiva constructivista. Al finalizar dicha enseñanza se evaluó a sendas divisiones, con la misma prueba. El grupo llamado B (39 alumnos) obtuvo calificaciones levemente superiores con respecto al grupo $\mathrm{A}$, dentro de los valores normales esperados.

A continuación, se eligió realizar en el período de síntesis temática ( 2 clases de 80 minutos cada una) la experiencia de confeccionar la red conceptual con el grupo $\mathrm{A}$ (33 alumnos), mientras el grupo B retomó el tema realizando problemas integradores adicionales.

La experiencia así planificada tuvo las siguientes ventajas:

- Ambos cursos tenían la misma docente.

- Tenían el mismo número de horas semanales para la asignatura.

- Tenían alumnos de nivel intelectual suficientemente heterogéneo dentro de un nivel sociocultural homogéneo.

- Habían recibido la misma enseñanza e idéntica evaluación.

A los alumnos del grupo A, se les informó que se haría con ellos un trabajo diferente y novedoso para evaluar la efectividad de un nuevo recurso didáctico. Esto los motivó y estimuló a participar activamente en la experiencia.

\section{METODOLOGÍA PARA LA CONFECCIÓN DE LA RED DE CINEMÁTICA}

La confección de la red conceptual con el grupo A fue un trabajo grupal que consistió en una serie de consignas:

* La primera consigna fue que los alumnos propusieran en voz alta un listado de los conceptos estudiados que, a su juicio, hubieran sido los más importantes. Así se confeccionó en el pizarrón, la lista de conceptos.

* A continuación, en la segunda consigná, se solicitó a los alumnos que eligieran pares de dichos conceptos y que armaran -por escrito- oraciones sencillas con ellos. Estas oraciones se leyeron y las más precisas fueron escritas en el pizarrón por la docente (respetando el diseño gráfico de la red planificada previamente por las docentes, pero desconocida por los alumnos).

* La tercera consigna, simultánea con el armado de la red en el pizarrón, fue que los alumnos discutieran la validez, precisión y coherencia de las oraciones propuestas (relaciones entre conceptos). La oración nuclear surgíó en cada caso como producto de una discusión y consenso de la clase.

Es muy importante destacar que los alumnos no propusieron en la lista de conceptos relevantes los específicos de la red \#1 (recuadrados en línea de puntos); esto era esperable por cuanto se trataba, justamente, de conceptos generalmente subsumidos. Los errores y las discusiones que surgían, al explicitar las oraciones nucleares para la red \#2 (básicamente la que surgió grupalmente), dieron cuenta de dichos conceptos inclusores faltantes 0 equivocados: las aclaraciones de la docente en el pizarrón fueron el bálsamo que hizo explícitas estas relaciones.

Lo más importante desde el punto de vista didáctico es que las explicaciones de la docente ocuparon el momento exacto para hacer eficiente la construcción del conocimiento en los alumnos; es decir, su intervención ocurrió a partir de la demanda que significaba tener a los alumnos argumentando a favor o en contra de conocimientos aún incompletos para ellos.

Poco a poco, el entusiasmo de los alumnos fue creciendo, la participación incrementándose y el debate entusiasmándolos. La docente señalaba incoherencias o generaba pequeños conflictos cognitivos para avivar las argumentaciones en favor o en contra de las oraciones propuestas. El timbre đe finalización de la clase implicó dejar para la semana siguiente la completación de la red con los conceptos aún sueltos.

Una vez confeccionada la red de cinemática hecha en conjunto por toda la clase, cada alumno la copió en su carpeta enterándose, recién entonces, que se trataba de una red conceptual.

Con estas actividades (confección de la red con el grupo A y la resolución de una guía adicional de problemas con el grupo B), finalizaron las clases del año lectivo 1991 . 


\section{Discusión de los resultados de la Experiencia I}

\section{Un nuevo instrumento didáctico}

E1 resultado más evidente fue la revelación de que la confección de redes conceptuales como actividad de cierre de un bloque temático es un nuevo instrumento didáctico de gran potencialidad.

Los alumnos se sienten motivados a participar en la construcción de las oraciones nucleares de la red. No temen equivocarse, pues ninguno de ellos debe repetir una definición de memoria, sino expresar con sus propias palabras aquello que han aprendido con algún grado de significación. Es un momento đe reflexión metacognitiva (Galagovsky 1993 b) muy importante que está netamente a cargo de los alumnos.

Un instrumento que ayuda al docente a evaluar las posibilidades cognitivas de sus alumnos

La confección de la red por parte de los alumnos fue un momento ideal para retroalimentar a la docente respecto del nivel de aprendizaje significativo que ellos habian realizado, pues se revelaron claramente tres categorías:

* los que habían comprendido el tema,

* los que habíar aprobado la evaluación, pero no habian comprendido cabalmente las relaciones fundamentales (muchos de estos alumnos desarrollan capacidades durante la confección de la red, y pasaron a engrosar la categoría precedente); y, finaimente,

* aquéllos cuyas posibilidades o intereses en el aprenđizaje fueron bajos. Este grupo fue irreductible.

\section{Conclusiones de la Experiencia I}

El alto nivel de comprensión logrado por los alumnos y la cantidad de errores que pudieron superarse -al volverse conscientes durante la explicitación de las oraciones nucleares- pudo haber sido suficiente evidencia cualitativa sobre la utilidad de este nuevo recurso metodológico que estábamos poniendo a prueba. Sin embargo, nuestra observación se prolongó en el año siguiente para evaluar el aprendizaje conceptual remanente en los alumnos tras tres meses de receso escolar estival.

\section{RELATO DE LA EXPERIENCIA II: EVALUACIÓN DEL APRENDIZAJE DE CINEMATICA LUEGO DE TRES MESES DE RECESO ESCOLAR}

\section{Una herramienta que posibilita el cambio conceptual en los alumnos}

Al inicio del ciclo lectivo de 1992 se avisó a los alumnos de los grupos A y B que se les tomaría una evaluación diagnóstica sobre el tema cinemática antes de comenzar el tema de dinámica (primero del bloque curricular del $4^{\circ}$. año del bachillerato). La evaluación se cumplió la segunda semana de clases. En sendas pruebas se permitió a los alumnos tener en el pizarrón todas las ecuaciones de los movimientos estudiados y la hoja de la red conceptual para el grupo A.

La prueba para el grupo A se dividió en dos ítems (Anexo I). El f́tem 1 apuntaba a averiguar sí recordaban todo el panorama de casos estudiados y factibles de aparecer en el tema de cinemática. En el ítem 2, al pedirles que propusieran un problema y lo resolvieran, intentábamos verificar pensamiento formal (Piaget 1976) y pensamiento reversible (Aebli 1987).

Las respuestas a los exámenes de ambos grupos tenían el mismo nivel de requerimiento en cuanto a contenidos y nivel de comprensión (Bloom 1966); sin embargo, la prueba para el grupo B podría contestarse, en alguna medida, desde algoritmos de resolución de problemas aprendidos de memoria, pues era un ejemplo absoluta-

Calificaciones obtenidas por los alumnos de ambos grupos sobre el tema cinemática.

\begin{tabular}{|l|c|c|c|}
\hline CALIFICACIONES & Ítem I GRUPO A & Ítem 2 GRUPO A & GRUPO B \\
\hline Bien resueito & $66,7 \%$ & $48,2 \%$ & $0 \%$ \\
\hline Parcialmente restrelto & $29,6 \%$ & $18,5 \%$ & $10,3 \%$ \\
\hline Mal resuelto & $3,7 \%$ & $18,5 \%$ & $2,6 \%$ \\
\hline Sin resolver & $0 \%$ & $14,8 \%$ & $87,1 \%$ \\
\hline
\end{tabular}


mente parecido a los problemas tomados en las guías de ejercicios desarmolladas durante el bloque temático a fines de 1991.

\section{Resultados de la Experiencia II}

Los resuitados de la evaluación diagnóstica se muestran en la tabla 1 .

A manera de ejemplo, presentamos en el anexo Il dos resoluciones del ítem 1 de sendas alumnas del grupo A: una calificada como «bien resuelto», y otra calificada como «parcialmente resuelto». Presentamos también en el anexo 3 dos ejemplos de los problemas propuestos por los alumnos para el ítem $2^{4}$.

\section{Discusión de los resultados de la Experiencia II}

El análisis de estos resultados indicados en la tabla I muestra espectacularmente el nivel de aprendizaje logrado por los alumnos. Entendemos que se trata de haber logrado un verdadero cambio conceptual en la estructura cognitiva de los alumnos por cuanto habían pasado tres meses de vacaciones desde el aprendizaje del tema.

El hecho de que ambos grupos contestaran la prueba con la posibilidad de consultar las ecuaciones, pero que sólo mayoritariamente los alumnos del grupo A las hayan podido aplicar correctamente, es la evidencia de que las respuestas involucraron altos niveles de comprensión y significacion de la trama conceptual correspondiente.

Este resultado tan contundente se vio reforzado por la prolijidad, clariđad en los gráficos, desarrollos y redacción demostrados por los alumnos del grupo $\mathrm{A}$, en contraposición con las tachaduras y rodeos en las escasas pruebas bien resueltas por los alumnos del grupo B. Entendemos que esto es un nuevo índice de la seguridad con que los alumnos manejaron los conceptos.

\section{RELATO DE LA EXPERIENCIA III: CONFECCIÓN DE LA RED DE DINAMICA}

Los datos presentados en el punto 4 nos motivaron a continuar con el uso de redes conceptuales para el tema de dinámica con el grupo $\mathrm{A}$.

Nuestro objetivo consistio, nuevamente, en enseñar en forma idéntica y desde un enfoque constructivista para ambos grupos, utilizando como cierre la confección de la red de dinámica con el grupo A y la resolución de una guía de problemas integradores adicional para el grupo B.

Esta vez, para poder concluir con mayor precisión acerca del valor de este recurso metodológico, no hubo un instrumento evaluativo diferente para cada grupo.
La red conceptual confeccionada previamente por Ias docentes

La confección de la red de dinámica (red \#3, ver página 349) nos demando nuevamente una reflexión metacognitiva sobre los conceptos substumidos que ocasionan errores en los alumnos. En esta oportunidad llegamos a Ias siguientes conclusiones:

* Habitualmente los alumnos presentan dificultades en Ia comprensión de que la dinámica incluye los conceptos vistos en cinemática, desde un modelo de situación más complejo, que tiene en cuenta la masa del móvil, y que estudia cómo influye sobre el movimiento de un cuerpo la interacción con otros cuerpos.

* Los alumnos tienden a generalizar la afirmación que dice:

"Si un cuerpo de mueve con velocidad constante, su aceleración es cerom, dejando excluido el caracter vectorial de la velocidad, que se hace explícito en el caso de movimiento circular uniforme, donde varía la dirección de la velocidad, y la aceleración es centrípeta. Es por este motivo que incluimos en la red \#3 los movimientos circulares -que se estudian con posterioridad a dinámica.

\section{Metodología para la confección de la red de dinámica}

Una vez conchuido el aprendizaje del tema, se solicitó a los alumnos del grupo A que se organizaran en grupos de 405 y armaran ellos mismos el listado de conceptos, las oraciones «precisas" y las organizaran Iuego en la red de dinámica, utilizando todos los textos y apuntes que quisieran. Al concluir la clase, la docente se llevó la red de cada grupo para su revision.

Tabla II

Calificaciones obtenidas por los alumnos de ambos grupos sobre el tema de dinámica.

\begin{tabular}{|l|c|c|}
\hline & GRUPO A & GRUPO B \\
\hline Total de alumnos & 37 & 40 \\
\hline Calificaciones* $\geq 6$ & $75,7 \%$ & $37,5 \%$ \\
\hline Calificaciones entre 4 y 6 & $18,9 \%$ & $40 \%$ \\
\hline Calificaciones $\leq 4$ & $5,4 \%$ & $22,5 \%$ \\
\hline
\end{tabular}

* Calificación máxima 10. 
La segunda clase se dedicó para discutir y consensuar las diferentes redes propuestas, organizando la versión definitiva de la red de dinámica en el pizarrón. El consenso colectivo demand6 argumentaciones y necesarias resig. nificaciones individuales.

\section{Resultados de la Experiencia III}

Finalizado este tiempo de repaso, ambos grupos fueron examinados con el mismo instrumento evaluativo que se muestra en el anexo IV.

Las evaluaciones para sendos grupos se tomaron en horas consecutivas, de tal forma que no hubo información entre ellos sobre el contenido de la misma. Las respectivas calificaciones obtenidas se muestran en la tabla II.

\section{Discusión de los resultados de la Experiencia III}

Nuevamente, la particular distribución de las califica* ciones evidenció un aprendizaje significativamente mayor para el grupo A. Asimismo, se reiter 6 el dato relevante de la gran prolijidad que caracterizó a las pruebas de este grupo.

Una variable que seguramente contribuy 6 a la obtención de estos resultados y que nos resultó inevitable fue que el grupo A había desarrollado una actitud positiva hacia el aprendizaje de la física y un interés en edescubrir» las oraciones nucleares que fue vivido como un motivante desafío intelectual.

También estimamos que el hecho de haber realizado un aprendizaje significativo del tema de cinemática constituyó un basamento más efectivo sobre el cual el grupo A construyo el tema de dinámica.

Otra observación importante fue que los integrantes más destacados del grupo A manifestaron una actitud autogestiva en la adquisición de los conocimientos de los temas subsiguientes.

Debido a esta confirmación acerca de los beneficios del trabajo con redes conceptuales, se decidió dar punto final a la investigación para poder utilizar este instrumento didáctico también con el grupo $\mathrm{B}$ en los temas curriculares subsiguientes.

\section{CONCLUSIONES}

La utilización de redes conceptuales, entendiéndolas desde la nueva propuesta que incluye aportes de la teoría psicolingüística para su confección, ha resultado ampliamente satisfactorio para el trabajo con alumnos.
Es necesario hacer, sin embargo, algunas advertencias:

* Las redes conceptuales sobre un dado tema, confeccionadas por distintos docentes o alumnos, no serán seguramente idénticas. Cada individuo podrá elegir, con un cierto grado de libertad, los conceptos que querrá rela. cionar. Lo obvio es que cualquier red debe mostrar afirmaciones siempre válidas al ser juzgadas por exper. tos en el tema.

* Con las redes conceptuales no se construyen concep-
tos. Por ejemplo, el concepto de aceleración no surgió a
partir de la confección de la red. La red no es una «varita
mágica», y su uso indebido será tan tedioso para los
alumnos como estudiar un capítulo del libro de
memoria.

Las redes conceptuales podrán utilizarse en momentos de reconciliación final de conceptos y como matriz organizadora de próximos conceptos, en el caso en que los alumnos estén familiarizados con su esencia.

Las ventajas evidentes de su utilización como instrumento didáctico resultan de:

* En primer lugar, al confeccionar previamente las redes, Los docentes deben efectuar una reflexión profun * da acerca de los contenidos fundamentales a enseñar y acerca de los conceptos explícitos y subsumidos del tema. Esta conscientización ayuda al docente a manipular con seguridad los factores generadores de errores en los alumnos, ya que, al construir la red con ellos, segu. ramente provocará el «afloramiento a nivel consciente» de los conceptos inclusores subsumidos, que podrán ser correctos o no.

* En segundo lugar, el ejercicio metacognitivo que realizan Ios alumnos al organizar las oraciones nucleares con los conceptos seleccionados es un sello que resignifica lo aprendido, dándole validez, contexto y precisión, a la vez que les permite revisar las conexiones conceptuales realizadas y concientizarse sobre los aciertos y equivocaciones desde una instancia autoevaluativa y autocrítica.

* En tercer lugar, es un instrumento que utiliza el lenguaje como una interfase de comunicación entre el contexto lingüístico vulgar y el vocabulario científico específico de la disciplina. Durante la discusión acerca de le elección de la oración nuclear apropiada afloran los conflictos semánticos sobre lo que los alumnos «dicen» $\mathrm{y}$ «aquello que querían decir».

En definitiva, la red conceptual no enseña por si misma, pero el tiempo de su confección es un momento de esfuerzo intelectual donde se resignifica y organiza conscientemente el aprendizaje. 


\section{NOTAS}

1. La teoría psicolinguística de Chomsky supone la idea de que todos los humanos heredamos la pauta específica de la especie de una gramática universal que nos permite traducir, reelaborar y codificar en oraciones nucleares a todos los significados de la información que recibimos bajo diferentes y ambiguas estructuras superficiales de lenguaje.

Chomsky sostiene que el receptor de una comunicacion debe ejecutar toda una serie de transformaciones mentales conscientes para asignar a la información que recibe una estructura profunda acertada, es decir, una comprensión de to que significa. La oración nucleares la representación linguística de dicha estructura significativa profunda.

2. La utilización del recurso informático donde se visualizan los móviles, los movimientos y la forma de sus gráficos también colabora en este sentido.

\section{REFERENCIAS BIBLIOGRÁFICAS}

AEBLI, H., 1987. Una didáctica fundada en la psicología de Jean Piaget, 20a, ed. (Kapeluz: Argentina).

AUSUBEL, D., NOVACK, J. y HANESIAN, H.,1978. Educational Psychology: A Cognitive View, 2a. ed. (Holt, Reinhart and Winston: Nueva York).

BLOOM, B.S., 1966. Taxonomy of educational objectives; The classification of educational goals. Handbook I: Cognitive Domain. (McKay Co.: Nueva York).

CHOMSKY, N., 1972. Language and Mind. (Janovich: EEUU).

GALAGOVSKY, L., VENDITTI, A., BIKEL, M., FLORES, M., CHERNIZKY, M. et al., 1990. Redes conceptuales: un excelente instrumento de comunicación entre docentes. Comunicación al II Congreso de Educadores en la Química de la Provincia de Buenos Aires. Argentina.

GALAGOVSKY,L., ROMERO, L., MÉNDEZ, M. y IERABEK, C., 1991. Redes conceptuales: una herramienta útil para la enseñanza de química. Noticias Panamericanas en Educación Química (F. L. Asoc. Químicas: Argentina), Vol. 3(4), pp. $2-4$.
3. Física. El Movimiento. Volumen 1. Prociencia. CONICET. Año 1988. Bs. As.

Cinemática 1. J. Abeledo y otros. Federación Universitaria de Buenos Aires.

Software Arandú: Newton I. Prof. Roberto J. Luis e Ing. Eduardo de Antueno. Rivadavia 822, piso 2. Buenos Aires, Argentina.

Castiglioni, Perazzo y Rela. Física I. Editorial Troquel. Buenos Aires. Argentina. 1990

Física. Lic. A. Chebli Murad. Curso preparatorio para la Universidad Tecnológica Nacional, regional Buenos Aires.

4. Cabe señalar que 10 de los problemas presentados fueron premiados incorporándolos en la guía de trabajos prácticos del año siguiente con los nombres de sus autores.

GALAGOVSKY,L.,1993a. Redes Conceptuales: su base teórica e implicancias para el proceso de enseñanza-aprendizaje de las ciencias, Enseñanza de las Ciencias, Vol. $1 \mathrm{I}$, pp. 301-307.

GALAGOVSKY, L., 1993b. Hacia un nuevo rol docente. Una propuesta diferente para el trabajo en clase. (Troquel: Argentina).

MOREIRA, M.A. y BUCHWEITZ, B., 1987. Mapas Concetuais. (Moraes: Brasil).

MOREIRA, M.A., 1988. Mapas conceituais e aprendizagem significativo, $O$ Ensino, Vol. $23, \mathrm{pp} .28$.

NOVACK, J., 1982. Teoria y práctica de la educación. (Alianza Universidad: España).

NOVACK, J. y GOWIN, B., 1988. Aprendiendo a aprender. (Martínez Roca: España).

NOVACK, I., 1992. Ayudar a los alumnos a aprender cómo aprender, Enseñanza de las Ciencias, pp. 215-228.

PIAGET, J., 1976. Le possible, l'impossible et le necessaire. Archives de Psychologie, Vol. XLIV. (Francia). 


\section{ANEXO I}

Evaluación diagnóstica para el grupo A:

Item 1: Graficar $\mathrm{x}=\mathrm{f}(\mathrm{t}) ; \mathrm{v}=\mathrm{f}(\mathrm{t}) ; \mathrm{y} \mathrm{a}=\mathrm{f}(\mathrm{t})$ para un movimiento uniformemente variado, considerando las diferentes posibilidades de signos.

ftem 2: Proponer y resolver un problema que incluya como datos los siguientes conceptos de la red: velocidad, aceleración, tiempo.

Evaluación diagnóstica para el grupo B:

El auto X está detenido frente a un semáforo. Se enciende la tuz verde y arranca con aceleración constante. El auto $M$ que viene (en el mismo sentido que $\mathrm{X}$ ) con velocidad constante, en un dado momento se adelanta al X. Un gráfico que representa la situación relatada sería:

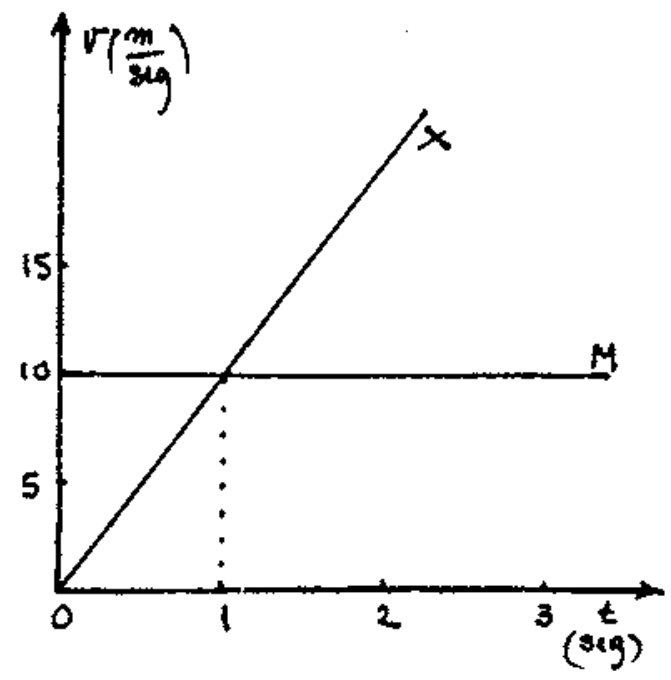

a) Confeccionar los gráficos superpuestos de la posición en función del tiempo para los alitos X y $\mathrm{M}$.

b) Cuántos segundos tarda $\mathrm{X}$ en alcanzar la velocidad de $\mathrm{M}$.

c) En qué instante alcanza $X$ a $M$ ?

d) Qué características tiene un movimiento uniforme?

\section{ANEXO II}

fiem 1:

a) Respuesta tipo, evaluada como «bien resuelta». (Cabe aclarar que la respuesta general fue del tipo cualitativo; es decir, no se pidió correlación de valores explícita entre los gráficos).
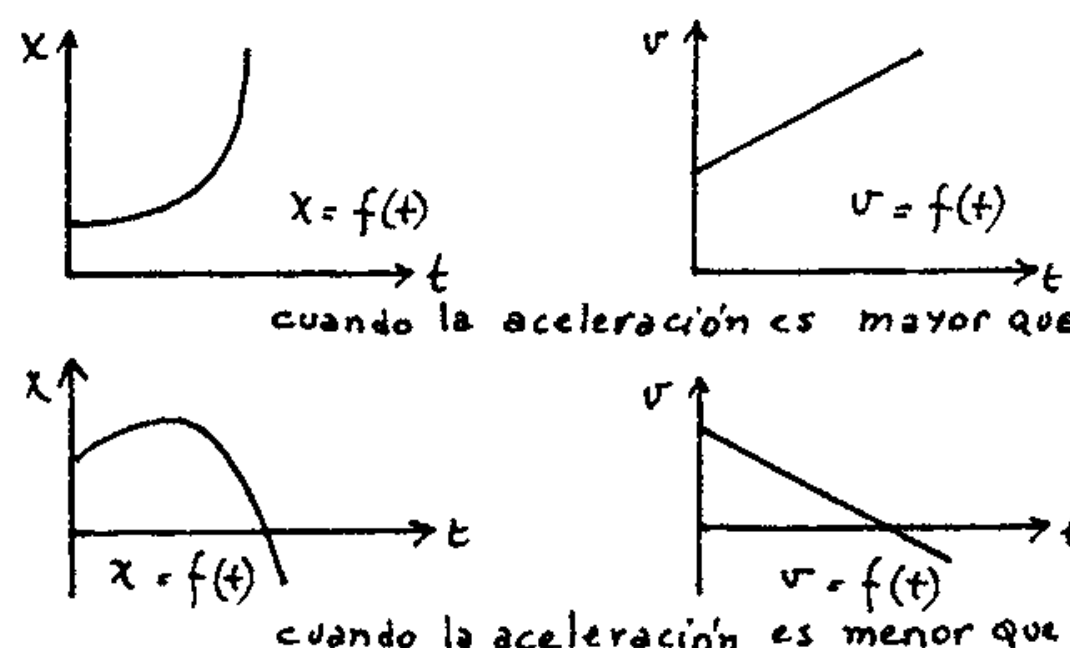

aceleración es mayor que cero
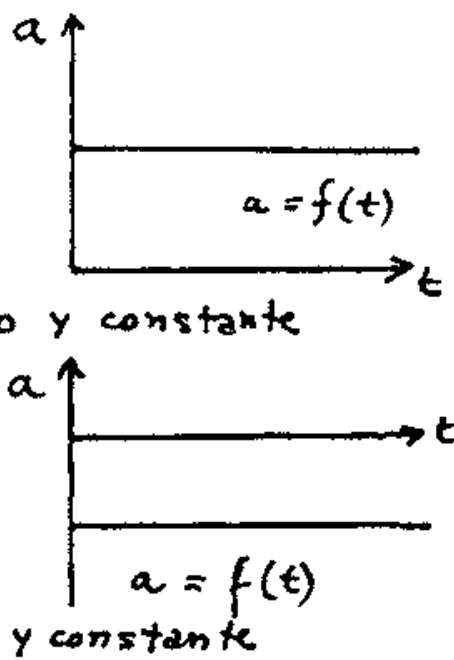
b) Respuesta tipo, evaluada como "parcialmente resuelta» pues, si bien incluye datos concretos y la expresión general de cada gráfica (ique no había sido pedido!), faítan los gráficos para el caso de aceleración $>0$.

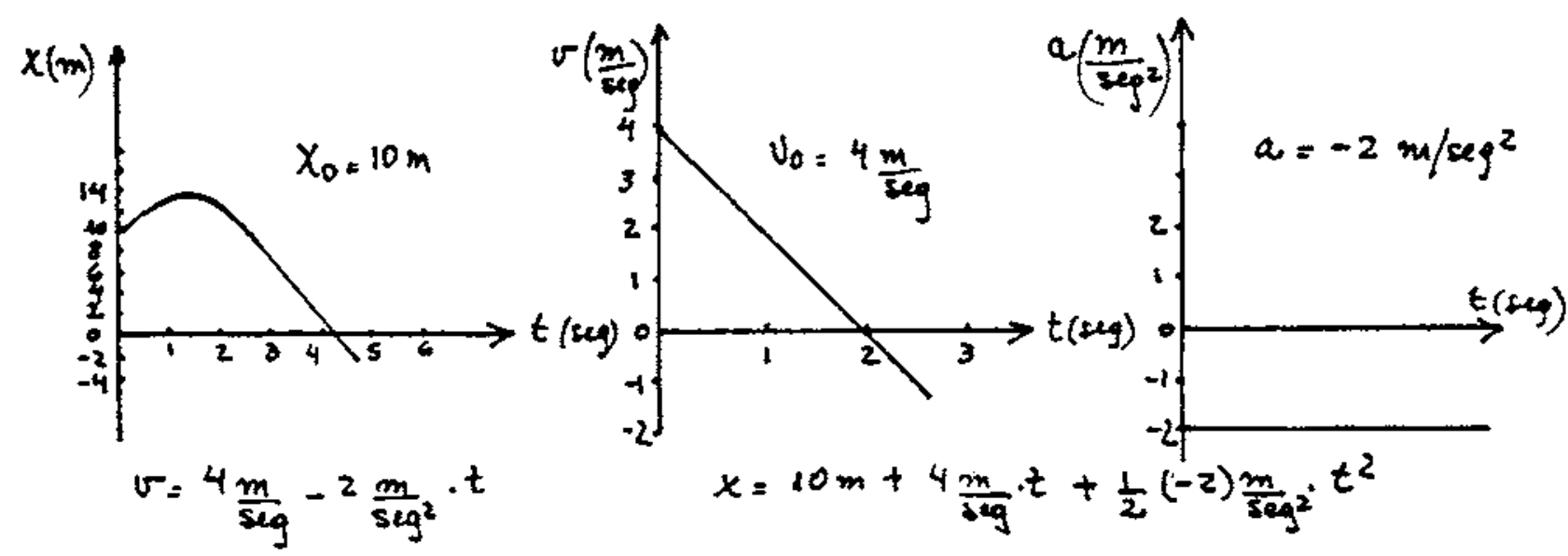

\section{ANEXO III}

Item 2:

I. Problema de Varesa $\mathrm{G}$.

Un gato circula por la calle con velocidad constante de $1,2 \mathrm{~m} / \mathrm{s}$, al pasar por un ábol sale en su persecución un perro que se encontraba descansando, el cual acelera uniformemente alcanzando una velocidad de $1,6 \mathrm{~m} / \mathrm{s}$ en $8 \mathrm{~s}$, continuando con dicha aceleración hasta alcanzar al gato.

a) Cuál es la aceleración que tiene el perro?

b) En qué instante logra el perro alcanzar al gato?

c) A qué distancia del árbol alcanza el perro al gato?

d) Hacer una secuencia gráfica a partir del enunciado.

II. Problema de Mariana $T$.

Un avión lleva una velocióad constante de $150 \mathrm{~m} / \mathrm{s}$. Al avanzar, entra en una zona de tormenta y, debiendo pasaria, acelera a razón de $10 \mathrm{~m} / \mathrm{s}^{2}$ durante $5 \mathrm{~s}$. Luego continúa volando con velocidad constante.

a) Graficar $v=f(t)$ y $a=f(t)$.

b) Hallar la velocidad del avión a los $4 \mathrm{~s}$ de encontrarse en la zona de tormenta. 


\section{ANEXO IV}

1. Un cuerpo de masa $m_{1}$ está apoyado sobre una mesa y unido mediante una soga a an segundo de masa $m_{2}$, como indica la figura.

a) Realizar el diagrama de cuerpo libre para ambos cuerpos.

b) Plantear las ecuaciones para cada cuerpo a partir de la segunda ley de Newton.

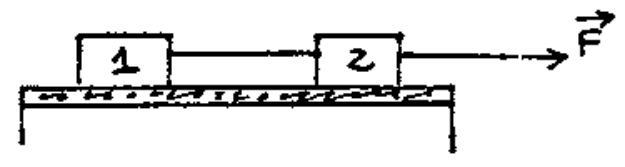

2. Un avión vuela horizontalmente con velocidad constante. Las fuerzas que actúan sobre él son: la fuerza motriz Fm, la de sustentación Fs, la resistencia del aire Ra, y el peso $P$.

Cuál de las opciones que se dan a continuación da una relación correcta entre las intensidades de esas fuerzas? Justifique su respuesta.
a) $P=F$ s y Fm=Ra
b) $\mathrm{P}<\mathrm{Fs}$ y $\mathrm{Fm}>\mathrm{Ra}$
c) $\mathrm{P}=\mathrm{Fs}$ y $\mathrm{Fm}>\mathrm{Ra}$
d) $\mathrm{P}=\mathrm{Fs}$ y $\mathrm{Fm}<\mathrm{Ra}$

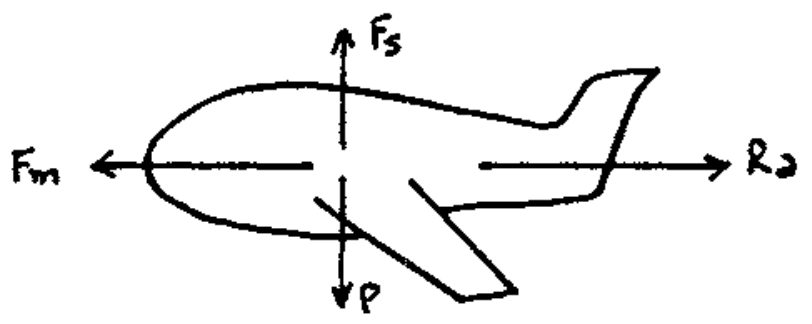

3. Un paquete cuya masa es de $50 \mathrm{~kg}$ es arrastrado por una persona mediante una cuerda que está inclinada $30^{\circ}$ con la horizontal, como indica la figura. Si el valor de la fuerza es $200 \mathrm{~N}$,

a) Indicar las fuerzas aplicadas en el paquete.

b) Determinar el valor de la aceleración.

c) Cuál es el peso de dicho paquete.

d) Qué movimiento le produce al paquete la aplicación de la fuerza $F$ ?

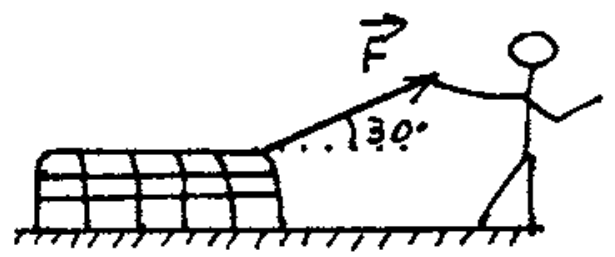


Red \#1 de cinemática

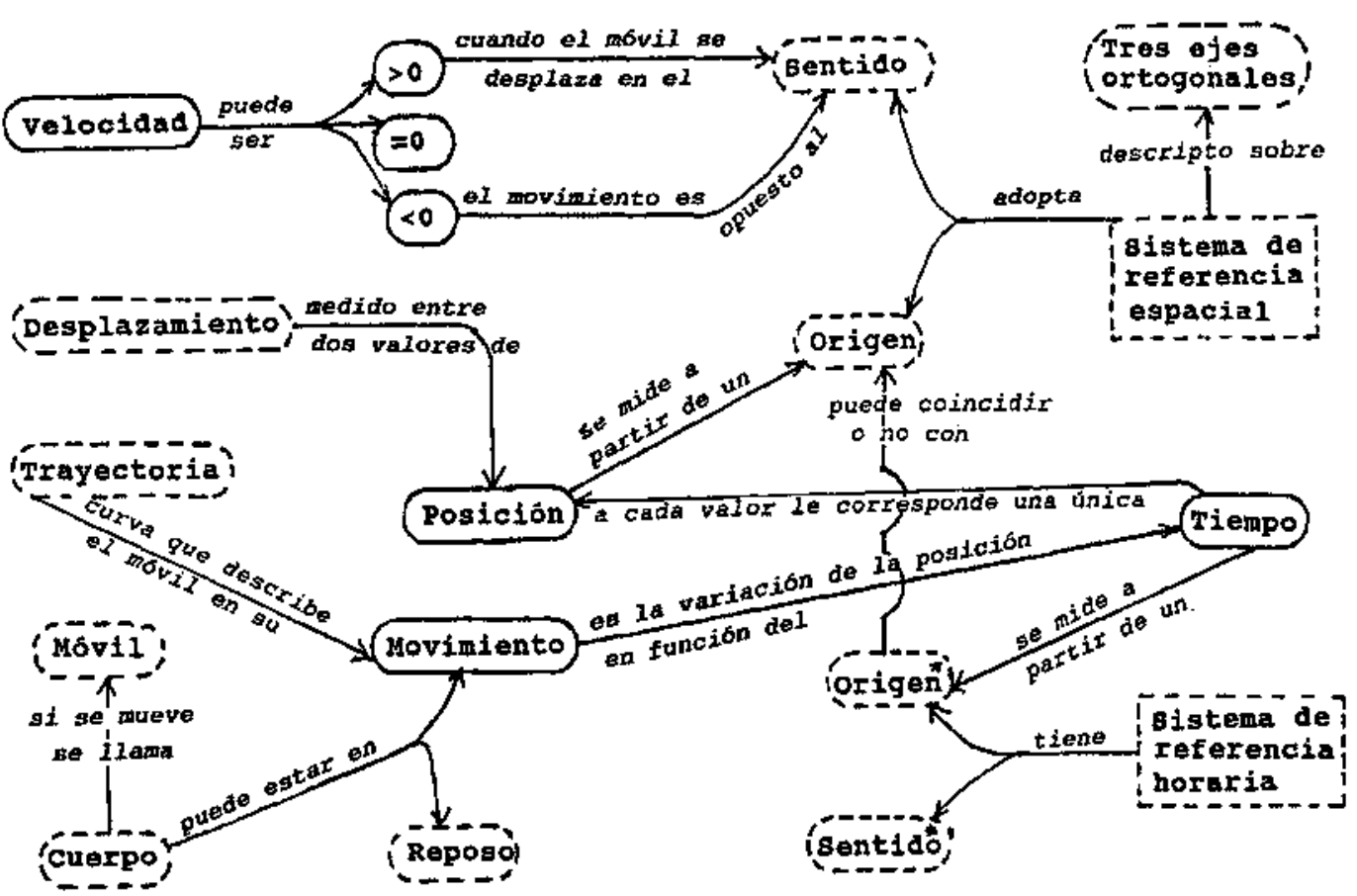

Red \#2 de cinemática

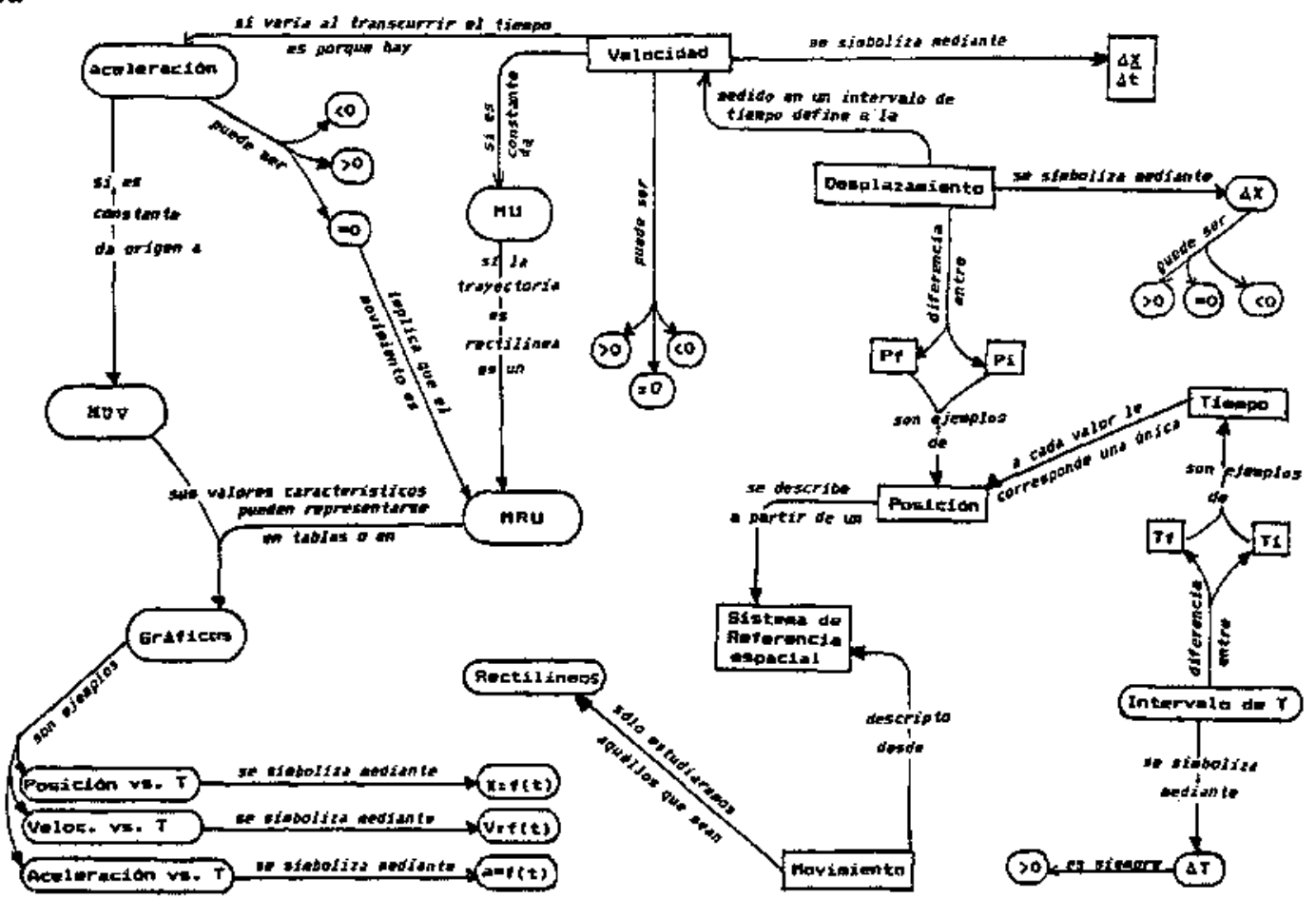

MU: movimiento uniforme

MRU: movimiento rectilíneo uniforme

MUV: movimiento uniformemente variado

Pí: posición inicial

Pf: posición final

Ti: tiempo inicial

Tf: tiempo final 
Red \#3 de dinámica

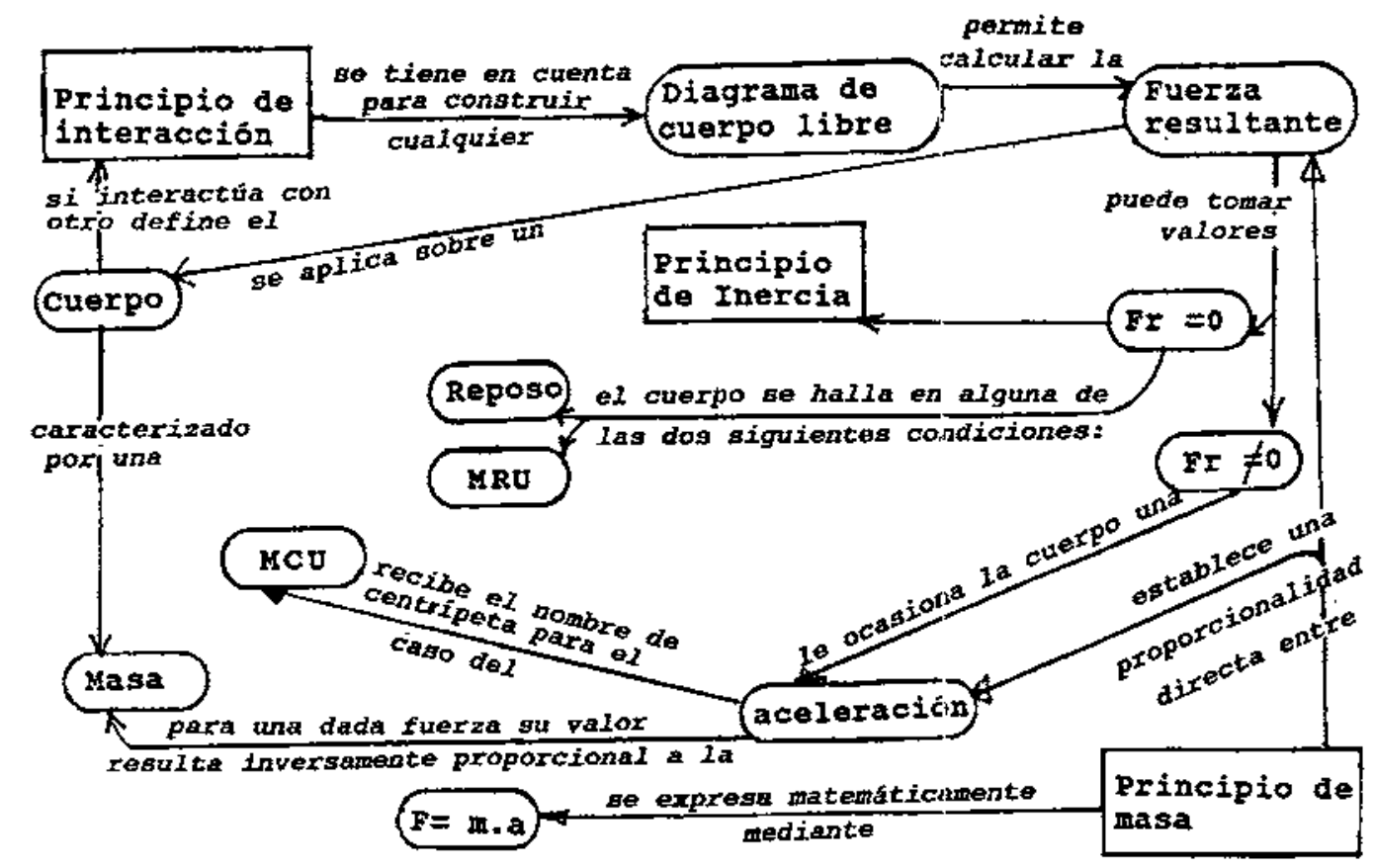

MCU: movimiento circulat uniforme 